\title{
Possible Adverse Event Attribution to Product or Procedure
}

National Cancer Institute

\section{Source}

National Cancer Institute. Possible Adverse Event Attribution to Product or Procedure.

NCI Thesaurus. Code C41359.

Possible adverse event relationship to study product or procedure is defined when there is less clear temporal association between product or procedure administration and adverse event, and other etiologies of the event are also possible. 